\title{
GOP Model: A Model For The Cosmos
}

Maria Zulmira Rodrigues*

Rua Almeida Garrett No 49, $3^{\circ}$ Esq. Pinhal Novo,2955- 127 Pinhal Novo, Portugal.

\begin{abstract}
This is a model for the Cosmos that makes it much less mysterious to our eyes. It is logical, simple and clear where the only exotic idea is the Big Bang, which is the founding idea on which it is constructed. This work was born of the idea that accumulating problems, in cosmology, is due to the absence of a suitable theoretical model. The advances in experimental cosmology are enormous. The information to be dealt with is immense, but it lacks this theoretical model consistent with the theories of physics. This is a widespread feeling among cosmologists. Because of the experimental successes, the theoretical work has not received sufficient attention. The independence of cosmology, as a science, has a price to pay, namely the theories and models used cannot be improbable. The models do not need large, or even any, theoretical justifications. What theoretical justification has Rutherford given, when he conceived a model for the atom similar to the solar system? They only need to be consistent with the facts and experimental data. In the same way, the dark matter and dark energy proposals to resolve some facts experimentally, cause us great perplexity. After decades of being proposed they not only have not given satisfactory responses but also created new problems. Furthermore, the dark matter and dark energy are two heavy burdens for physics. They are not consistent with the canons of scientific theories. These must be as simple and beautiful as possible.
\end{abstract}

Keywords: GOP model; Great primordial wave; Inner universe; Exterior universe

Abbreviations: GPW: Great Primordial Wave; GOP: Grande Onda
Primordial

\section{Introduction}

The dark matter and dark energy are two heavy burdens for physics. They are not consistent with the canons of scientific theories. These must be as simple and beautiful as possible. A theory that introduces an immense confusion and an accumulation of problems is not simple or beautiful. These and other issues led us to seek another model for the Cosmos because we have the strong conviction that something is not right in all this. In this quest we have decided to go a different path from the one that has been adopted up to now - to look for a solution for every problem - which has proved ineffective. We decided then from the knowledge that we consider more consistent without worrying about the resolution of concrete problems: we accepted Big Bang as a founder idea not only by self-conviction but also because the facts have given it more credibility. This has been a singular moment, and there is strong evidence that something similar has happened. This led us to the idea of a primordial atom, whose nucleus is very massive and with great stability, but where instability can happen and give rise to a tremendous nuclear explosion.

We have accepted a universe solely consisting of common matter, because until today nothing different has been found. Being this a model for a large-scale structure we have adopted Newtonian mechanics and its mathematical formalism for the interpretation of observational data and formulation of hypothesis not requiring, therefore, no new or more sophisticated formalism. This formalism has been shown consistent with many observational data. It will be with this mathematical formulation that we should look for an explanation for the experimental values, for the issues such as: cosmological constant; Accelerated expansion. In the same way to try to characterize the gravitational field created by GPW in the IU, which may allow a better knowledge of all the phenomena influenced by gravity. The model we conceived is a revolutionary model, being different from all the existing proposed, and it is consistent with the ideas defined by Stephen Hawking and others. The models must be simple, to conform to the known facts, experimentally testable and allow hypothesis formulation. This model has all of this. With the model conceived we evaluate a large group of the current and most enigmatic phenomena on the Cosmos (dark matter and energy, accelerated expansion, filaments, cosmic inflation, background cosmological radiation, ...) and, for each phenomena, we present hypothesis and conjecture compatible. The fact that the model responds astonishingly to all the issues tested has encouraged us to divulge it hoping that experts from the different areas of cosmology evaluate it in a finer way.

This work has been endeavored, due to great perplexity caused by the hypotheses of the existence of dark matter and of a dark energy proposed to solve some experimentally found facts. These facts became mysterious problems, because these concepts haven't led to satisfactory answers, and in many cases, they gave origin to new problems. We must add that the dark matter and dark energy are two heavy loads for Physics. Theories should be as simple and beautiful as possible. The introduction of these two concepts has caused a great confusion, an accumulation of problems and, likewise, we cannot say that such a mysterious and incomprehensible matter may be something beautiful. If for us to understand about $4 \%$ of conventional matter the task seems endless, what kind of battle would we have to face, bearing in mind that more than $90 \%$ of the matter can't be seen, can't be felt and that it practically doesn't manifest itself [1-3].

These questions lead us to search a model for the Cosmos. Thus, we consider, with conviction that something wasn't correct in all this.

*Corresponding author: Maria Zulmira Rodrigues, Rua Almeida Garrett No 49 $3^{\circ}$ Esq. Pinhal Novo,2955- 127 Pinhal Novo, Portugal, Tel: 919059702; E-mail: azulrodrigues@sapo.pt

Received November 08, 2017; Accepted December 11, 2017; Published December 19, 2017

Citation: Rodrigues MZ (2017) GOP Model: A Model For The Cosmos. J Astrophys Aerospace Technol 5: 153. doi:10.4172/2329-6542.1000153

Copyright: (c) 2017 Rodrigues MZ. This is an open-access article distributed under the terms of the Creative Commons Attribution License, which permits unrestricted use, distribution, and reproduction in any medium, provided the original author and source are credited. 
Based upon this conviction was born the proposal of the model, which we now present. One can also mention that the Big Bang, as a founding principle, is the idea that also has, with conviction, the greatest acceptance in the Scientific Community [4-12]. This model, as we will further be able to see, finishes with the above-mentioned burden.

\section{Development of the Model}

GOP model is a model for a large-scale structure, the Universe, and is part of the idea that this is constituted only by the conventional matter, because until today no other kind of matter has been found. This idea is also strengthened by the persistence of the law of conservation of the matter. Newtonian mechanics was adopted and its mathematical formalism for the interpretation of observational data and formulation of hypothesis, consistent with the model, not requiring large-scale explanations of any new or more sophisticated formalism. This formalism showed to be consistent with many observational data. It will be based in this mathematical formulation that we should look for an explanation, through the experimental values, for the issues such as: Cosmological Constant; Accelerated Expansion; ... Also in the same way to try to characterize the Gravitational field created by Great Primordial Wave in Inner Universe, which could allow a better knowledge of all the phenomena influenced by gravity.

This paper, beyond the description of the model, also addresses a large number of cosmological problems which does not permit a mathematical or a more developed approach of each of them. This will have to be done by specialists in each area. As an example, the Cosmological Constant, that was treated with the Newtonian formalism appears related to an energy [13], which is in agreement with this model. The Cosmological Constant is in this model associated with a potential gravitational energy and not to any completely unknown grandeur and isn't a constant.

It is taken for granted that we accept that our Cosmic Era began with a colossal explosion, colloquially called the Big Bang (BB). Likewise, in our dimension, we know that the biggest explosions are those caused by the atomic bomb. It is from them that we are going to extrapolate, to concept our model [14]. These explosions have origin in reactions between elementary particles of matter. This fact suggests something primordial which strengthened our conviction that this inspiration was acceptable.

As a start, we are going to observe some images of atomic explosions. These images have marked our recent imagery and it is known that in the moments following the great explosion there appears a cloud with the shape of a mushroom. Looking more attentively we can see, that in general, surmounting this cloud, others more diffuse appear (Figure 1). This type of deflagration happens under the Earth constraint and it presents an axis of symmetry, which passes by the hypocentre and is perpendicular to the Earth. Extrapolating to the BB situation, without this constraint, and doing a rotation using the constraint line as axis, we transform this axis of symmetry into a plane of symmetry, the mushroom cloud into a big and massive circular wave, externally surrounded by another more rarefied, staying a flat zone. It is from this above presented idea that we are going to build our model (Figure 2). It these model, these three zones that we referred to will be called: Inner Universe (IU); Great Primordial Wave (GPW); Exterior Universe (EU).

The IU, which results from the rotation of the chimney, is constituted by galaxies, nebula, ..., stars. This zone of the Cosmos is flat, it is situated on the plane of symmetry, that passes by the BB and that coincides with the zone of largest intensity of the gravitational field of the GPW. All the space of the IU, in under the action of the gravitational field of the
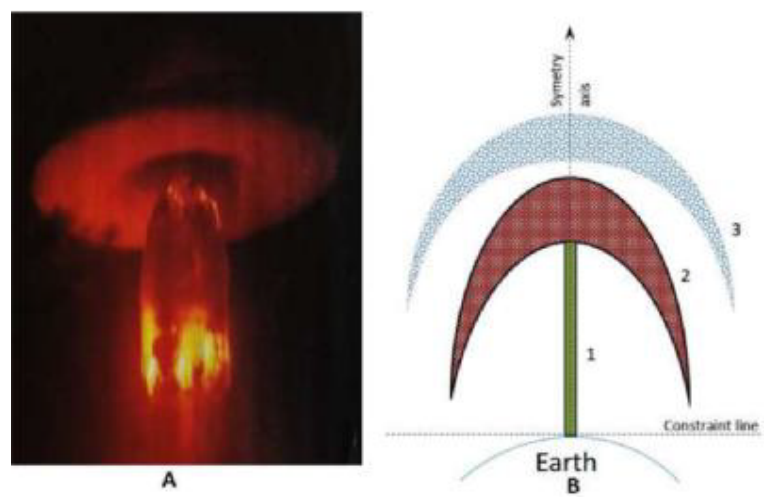

Captions: 1-Deflagration cannon; 2-Mushroom cloud; 3-Upper cloud. Figure 1: A-Photo of an atomic deflagration. B-Schema of an atomic deflagration.

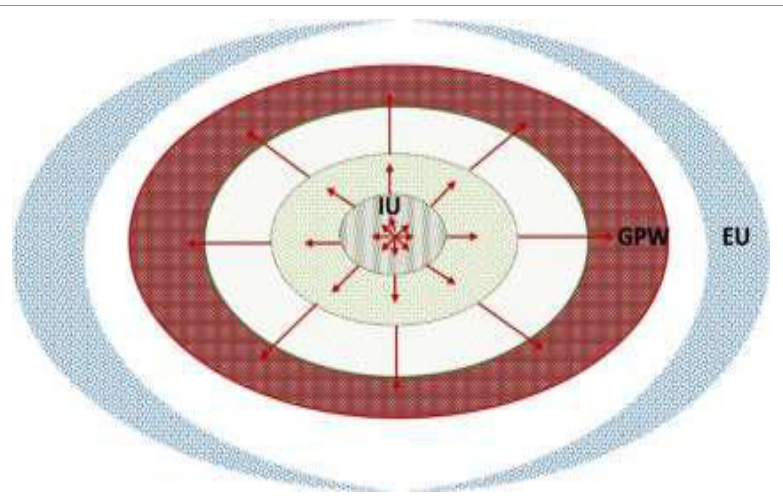

Figure 2: Schematic representation of GOP model for the Cosmos.

Captions: IU-Inner universe; It is a result from the cannon's rotation; GPWGreat Primordial Wave; It is a result from the mushroom's wave rotation; EU-Exterior Universe; It is a result from the rotation of the upper zone of the mushroom.

GPW. Thus, any being of this universe is subject simultaneously to the local field, and immerse in the field GPW. It is on this flat zone that our Observable Universe (OU) is situated. This one, isn't, probably, in the Center of IU and, therefore, the forces of the field GPW, in our universe are not uniform in its border. One zone of this border, nearer GPW, is subject to more intense gravitational forces than the opposite zone.

The GPW, which results from the rotation of the mushroom wave, is an immense wave of matter, interiorly concave, which involved the IU, forming a strong barrier, which separates it from the EU.

This above-mentioned wave, which is about $90 \%$ of the matter of the COSMOS, creates a gravitational field in IU that increases from BB towards the wave. This gravitational field is very strong in proximity of this wave. The EU, which results from the rotation of the upper zone of the mushroom may, probably, be the region of the Cosmos where the anti-matter dislocated itself, as consequence of some slight difference, which exists between it and the matter. If the EU is constituted by anti-matter in a situation of low density and temperature that may be in a primordial and little developed state. This idea is suggested by extrapolation of what happens with the atomic bomb, where over the mushroom wave another more diffused one is formed. This region is constituted by particles, which acquired a greater speed. Due to the mysterious absence of anti-matter in our universe, we can think that, at the formation moment, the slight difference between them, for example 
the temperature, makes the anti-matter advance, overcoming the GPW barrier, and, in this way, being definitely away from IU.

This is the Cosmos model we propose, inspired, and having as a principle the idea of a tremendous explosion, in a unique moment, denominated by BB. In this model the BB won't have had the origin in an incandescent and chaotic ball of high entropy, but on a radial explosion, giving origin to a Universe with more symmetry, and, consequently less entropic.

Our Cosmos is now an immense flat zone, denominated IU, where our OU is situated, surrounded by a colossal massive wave denominated by GPW and, having externally, a diffuse and rarefied zone called EU (Figure 3).

\section{GOP gravitational field in the inner universe}

Let us look attentively at the IU, as it is from it that we observe and think about the COSMOS, and we are going to reinterpret phenomena we know in the light of this model. Let's start by analyzing the gravitational field of the GPW in the IU, as we consider this of primordial importance. This field isn't constant, being zero in BB and increasing from there towards GPW, from a certain point onwards becomes dominant in relation to the local field.

Since its origin is not known, the gravitational field created by the GPW in the IU, led us to think it to be a stranger anti-gravitational field as the line forces start from a point - BB - and then they go towards the infinite.

It is exactly the contrary of what we are used to see with the material bodies of our material universe, the lines of force go towards the center of the mass of the body, i.e., towards a point (Figure 4).

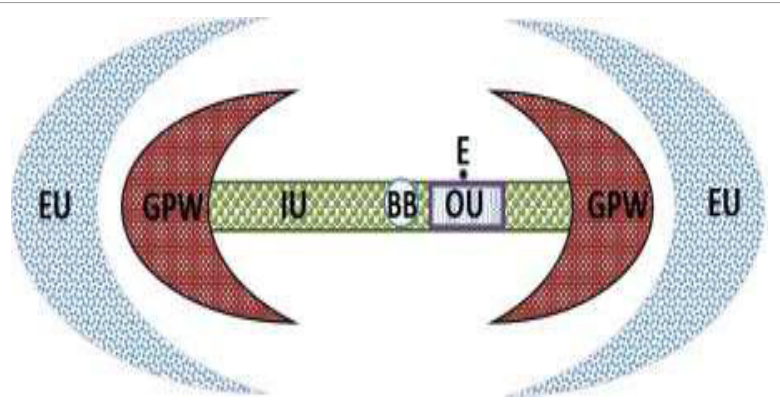

Figure 3: Transversal cut in GOP model for the Cosmos passing by BB.

Captions: IU-Inner Universe; BB-Point of origin of the Big Bang; T-Planet Earth; OU-Observable Universe situated in IU; GPW-Great Primordial Wave, an extremely massive wave, which involves all the IU; EU-Exterior Universe, Very extensive zone, with low density and low temperature.
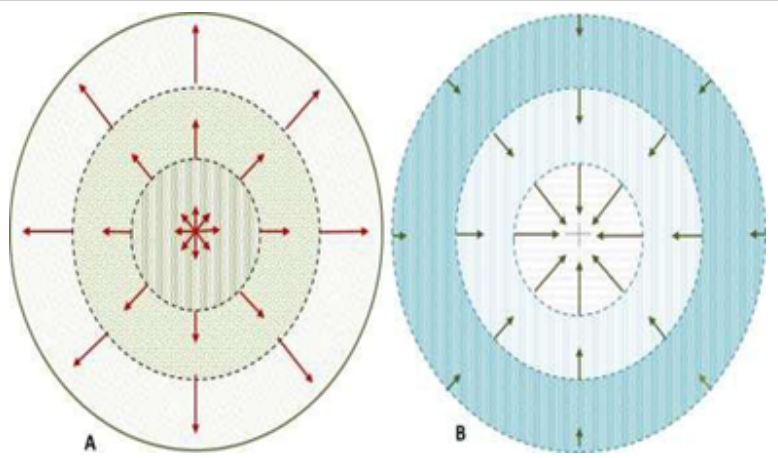

Figure 4: A-GPW Gravitational field in inner universe (IU), B-Gravitational field of the earth.

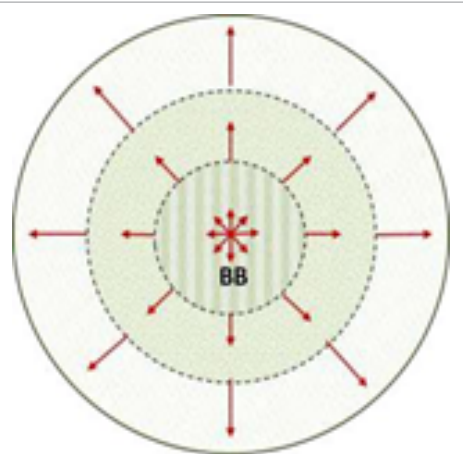

Figure 5: GPW: Gravitational field in inner universe (IU), according to the plan of longitudinal symmetry.

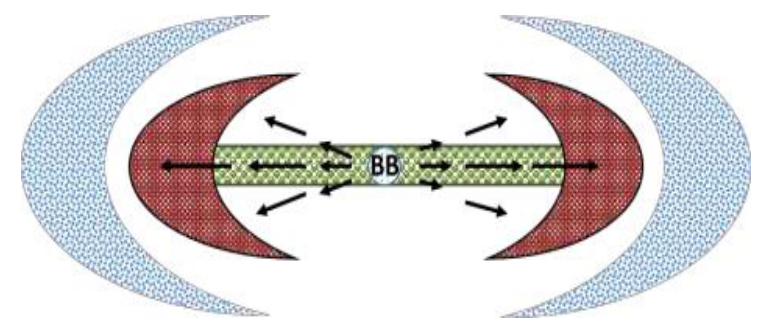

Figure 6: GPW: Gravitational field in the plan of transversal symmetry.

All this led us to the tremendous today's confusion. The dark matter (DM), i.e., missing mass, is no more than the concentrated conventional matter in GPW and the strange anti-gravitational forces are simply the forces of gravitational field of this immense wave (Figure 5 ). The IU is situated in the plane of longitudinal symmetry (that passes through $\mathrm{BB}$ ), and it is the region with the biggest intensity of this gravitational field. It isn't very likely that our OU is in the center of the IU, and so, there is a region of this universe that is near GPW. This region is, therefore, attracted by more intense forces.

Observing from the Earth and looking far away, all the bodies seem to be distancing themselves in an accelerating way $[15,16]$. In this model to explain the strange gravitational effects observed in galaxies, the hypothesis of DM is no longer necessary as they may be easily explained by the action of the conventional matter of GPW. This is a region of our OU, which seems to be attracted to a far distance, with a greater intensity. This phenomenon, which has been interpreted, with the hypothesis of a "Great Attractor" can, after all, not be more than the proximity that exist between that zone and GPW. The same happens with the deviation to the red of the super-new, the accelerated distancing from the "clusters", and from many other phenomena, which are considered mysterious [17]. On the opposite side of OU, those forces are weaker.

If the Cosmos is situated very far beyond our OU, and accepting as a principle the $\mathrm{BB}$, we must believe that something happened at a much faster speed than the one of the light in vacuum. Nothing tells us that this doesn't happen today, in the border with GPW. In the zone near GPW, the velocities may be so high, that the laws we know are not applied. If you observe from any place in the IU, and in any direction, the bodies are in accelerated movement. The greater the distance the bigger the acceleration. From a certain point onwards the bodies, inexorably, are going to dislocate themselves towards GPW. If GPW is still in expansion then the IU is becoming more rarefied, both by the increased interior space, and by the fact that the GPW continue to attract and capture matter. The gravitational field, in the transversal 


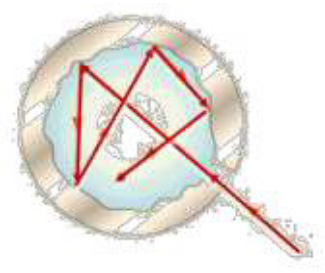

A

Figure 7: A-Black body, B-GPW universe
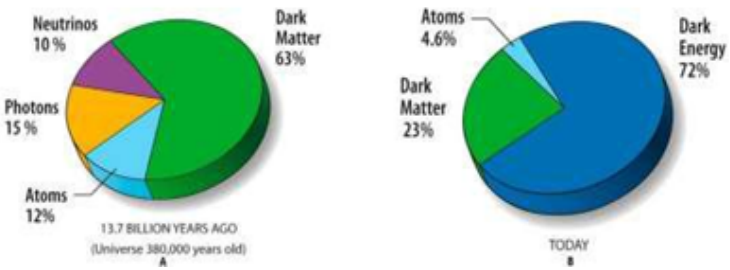

Figure 8: Cosmos composition: A-13.7 Billion years ago, B-Today.

plane has a different image. The forces have a bigger intensity in the plane of the IU and they go decreasing, being minimum, or null, in the perpendicular direction (Figures 6-8). The result of this forces is always situated on the plan of the IU. This can be a justification for the flat or ellipsoid form of most of celestial structures.

\section{Testing the Model}

We are going to test this model with the biggest number possible of the mysterious problems that are to be solved, and, also, with known facts. We are going with the most controversial questions that led to the formulation of the hypothesis of the existence of DM and Dark Energy (DE). In 1929 Edwin P. Hubble announced that, except for the group of galaxies (Local Group), to which the Milky Way belongs, all the others are moving away. This discovery led to the idea that the Universe is in expansion. This fact is also considered as an evidence of the validity of the theory of BB. Studies with supernovas done from the "Hubble Space Telescope" (HST), 1998, confirm and refine this expansion.

Also, the study of the rotation movement of the galaxies revealed that these have higher speeds than expected, due to the amount of matter that they possess. The amazing velocities of stars that are situated in exterior regions cannot be explained by the existing matter. We need a much higher quantity of matter to respect the laws of Newton. It is the "Missing Mass" problem. So, as to explain these strange facts, we proposed the existence of a hypothetic form of matter and energy that start to be known as DM and DE [18]. The scientists proposed that each galaxy lived inside a halo, not yet detected, of DM, which would exert a sort of anti-gravitational action, accelerating the exterior orbits.

If these strange facts were not known, they could have been foreseen by the GOP model. With this model the explanation is simple: The greater the distance from the center of the mass of the galaxy, smaller is local gravity; thus, the GPW field assumes more preponderance and provokes an acceleration opposite to the local field. Accelerated expansion is also, as we have already seen, explained by this model. These facts have now logical and plausible answers, based on a model that doesn't introduce any mysterious rule beyond the BB. Therefore, you can't say that it substitutes one mystery by another. Will it be, that by eliminating these phantasmagoric presences that, until today, have not yet solved any problem, having on the contrary created many more will everything become less mysterious?

\section{Testing another's problems connected with dark matter and dark energy}

Different acceleration between clusters of galaxies or galaxies in the same cluster: Another mysterious fact is the different behaviour of $\mathrm{DM}$ and of DE on "clusters" or individually on the galaxies of the same cluster [19].

The given explanation is that the $\mathrm{DE}$ is clearly selective when it looks at celestial bodies. It affects with a different degree (big or small) according to the dimension of the body. It causes positive acceleration among clusters of galaxies, but not among galaxies of the same cluster [19]. This is an explanation where the DE doesn't solve the problems to which it is proposed, and it even creates some more. It is very difficult to accept this capacity to discriminate the bodies, by dimension, acting in different ways. It seems too strange, even to be accepted as a conjecture. With the GOP model we easily find an explanation: In the clusters of galaxies, where the local gravity keeps them united, they will dislocate together, but, in the clusters, distant one from another, the gravitational attraction among them is weak, and doesn't manage to overlap the GPW field. The domination of this field leads to the separation of the clusters, with positive acceleration.

Filaments and walls of dark energy: These filaments are long and fine structures, like wires. The walls are more extensive and flatter structures than the filaments. Transversal cuts show flat sections, where can be defined a bigger and a smaller axis [20,21]. The Walls and the Filaments are interconnected and forming a mesh. The attempt to explain these strange Filaments and Wall recurring to the DM wasn't very successful, like all the problems where this concept is used. Until today none of this is clarified, and, in general, these attempts, cause new problems to appear. In 2005 only in the dark matter field, did eleven new unexplained phenomena appear, resulting from these attempts [19]. However, when using GOP model, a very clear explanation appears: These Filaments and Walls are composed of matter that dislocates itself, according to the line of forces of the GPW field. If the filaments, eventually, approach a group of galaxies they can be attracted by them. This must be a generalized event, making a mesh appears, connecting all (or many) groups of galaxies. This mesh is formed by interconnected walls and filaments. We can, probably, observe a direction in the flow of this mesh because it is surely heading to GPW. Knowing this we may obtain some information about the lines of the GPW field and even determine from where they start, i.e., the place of $\mathrm{BB}$, and where they are going to, i.e., GPW. The "Sloan Great Wall" (SGW) discovered in Princeton in 2003, 1.38 billion light years and is away from Earth, approximately, 1 billion light years. It has been suggested that SGW was constituted by the alignment of three filaments and not by a structure itself.

With the GOP model, this seems natural, in relatively near regions, the field lines may be considered almost parallel, being possible that the filaments also present parallel routes, that if observed in certain directions, seem to overlap. Once again, the GOP model shows coherence with the facts in opposition to what happens with the dark matter.

Energy of vacuum: The Energy of Vacuum (EV) is presently considered as a possibility for the attributed effects to the explanation for DE. If the concept of EV is born to explain them, according to the GOP model, it is natural that this doesn't lead to a great advance in the explanation of the phenomena of the COSMOS, as it is a concept that is born to explain another one that doesn't have any reason to exist. And it is the reality that confirms it, as the found values through this hypothesis are completely in disagreement with the experimental 
ones. The results obtained in this both ways are considered completely senseless. In the primordial Universe, the EV was negligible in comparison with the matter, but recently the situation was reversed and the EV began to dominate [22]. In the GOP model, the variation of EV has started to dominate. In the GOP model, the variation of the DE corresponds to the variation of the GPW gravitational field. Initially this field was weak inside the IU, since GPW ray was small and, therefore, the interior forces practically annihilate one another, and are null in BB, but as this radius increased GPW field forces began to dominate in certain regions of the IU.

In fact, in the early universe the EV was negligible in comparison with the matter while recently the situation was reversed, and the EV began to dominate. With the GOP model the variation of EV is the gravitational field of GPW. Initially this field was weak inside the Inner Universe, due to the fact that the GPW ray was small, and, therefore, the interior forces very weak, but as the ray increased, the GPW field forces began to dominate in certain regions of the IU near GPW.

Collision of galaxies: To accept the existence of halos of DM, surrounding the galaxies, when you intend to interpret the collision among them seems to be a difficult task. If the presence of DM serves to interpret the immense acceleration on the border of galaxies, then, how can this same matter allow collision between them? And why the galaxies of the same cluster don't increase the distance between them? The given explanation seems to be rather obscure. Let's see: the DM has the capacity to distinguish the cosmic entities, according to the mass that they possess and acting in a different way, in relation to the high or low value [23].

That strange hypothesis of a DM in the space of galaxies that prevents them, from collapsing, would never allow, for the same reason that two galaxies would collide. Andromeda and the Milky Way wouldn't be in collision if it were like that. But even stranger is this capacity to act in a radically different way, according to the mass of cosmic structures. Another strange characteristic to add to so many others, is the fact that a matter able to do so many things can't be found. In 2007 "Abell 502" represents a collision between galaxies of the same dimension. This collision behaves in a different way from the one described in 2006, which gave origin to the "Bullet Cluster" created by the passing of a little massive cluster, trough one of the big dimensions, with the velocity of $4500 \mathrm{~km} / \mathrm{h}[19,24]$. In the case of "Bullet C.", each of the original clusters, in collision, kept their identity even after the crash was complete [25]. Now the "Abbel" collision, created by two clusters of equal dimension, which collide at low speed, shows that there has been an interaction. The after collision aspect attests it, by showing a new cluster [26]. If one explains these collisions, admitting the existence of DM it seems to be complicated, wherever accepting the GOP model, everything seems to be is easier. With this model, all the process is simply an interaction in the common matter. The GPW field acts on the less massive galaxies (weak local field) causing a higher velocity than the one on the more massive galaxies (strong local field).

\section{Testing other problems}

The great attractor: The Local Group, of which the Milky Way and Andromeda are part, is moving towards the super-cluster of Virgo (which contains thousands of galaxies) at a velocity $\sim 250 \pm 50 \mathrm{~km} / \mathrm{s}$ $[27,28]$. Clearly this velocity cannot be attributed to the action of Virgo.

Recent studies about the peculiar movement of galaxies show that it is coherent on a large scale that the Local Group moves in mass, towards the cluster of Hydra and Centaurs and all of them are being pulled by the Great Attractor (GA) or, probably by the effect of a concentration of clusters. They move at about 1.5 million km/h. In 1986 it was determined that it is found at 250 million light-years of Milky Way in the direction of Centaurs. The GA has the length of 400 million light years. The Super Cluster Shapley, more massive than the GA, is found at 700 million light-years in the same direction of Centaurs that pulls us and the GA [29]. Today Harley Shapley, at 650 million light years from Earth, is the most massive structure that is known in our OU. It is the "Attractor" to all the galaxies of our neighborhood.

The tendency of galaxies to move in a certain direction, which was thought to be towards GA, is speculated today in 2008, to be out of the OU. The GPW can be the candidate to GA, as our Universe has, probably, a nearer zone to this wave and it is in that direction that everything dislocates itself more rapidly [30].

Cosmic inflation: In the GOP model a certain type of inflation is acceptable and predictable. This begins in a short initial moment, which followed the $\mathrm{BB}$, where the phenomena were extreme. The appearing of the IU follows with a high density of matter and, consequently, an intense local gravity that competed with the still incipient GPW gravity, due to its small ray, having consequently a slowing down of the initial expansion. At the beginning of the Cosmic Era the flow of matter of the IU to GPW, due to the strong local gravity, was weak but continuous, which, allied to the increasing of the GPW radius, weakened the local field by passing the GPW field to predominate in certain zones. It was the beginning of an accelerated expansion of great part of matter in the IU in direction to GPW. If GPW continue to increase it will lose leadership in the IU, not capturing matter anymore and cooling until it stops. The IU may know some stability with much less matter and more extension, having not any possibility to attracting GPW. The anti-matter will expand itself, colder and colder, and more and more rarefied, without evolving. As it is very rarefied, the possibility of reacting with GPW is low. The IU will also stabilize [31-33]. It is, probably, what will happen to the Cosmos if this model is adequate?

Cosmological coincidence: Cosmological coincidence is a problem. Today's density of the matter and density of cosmological constant are the same order of grandeur, indicating that DE started to dominate today's Universe [22]. Today it is considered strange that there is a coincidence between the density of matter of the Universe and the VE [34]. In GOP model that is stranger, as VE isn't but a gravitational potential energy, and this is, surely connected with density of matter of the universe. In GOP model this is not strange, as it is logically expected. In this model the relative balance between these energies varies rapidly as the IU expands. The density of the matter is initially elevated in the IU and the field GPW weak but, with the expansion of this, the density of the IU decreases and the GPW field increases starting to dominate in a large part of the UI. The balance between these two fields will create conditions of great stability in certain regions for the emergence of life. A part of our OU may be in these conditions. This stability has been progressing in the IU from BB, and eventually when GPW stops, already sufficiently cold, and without matter within its reach to attract, maybe the IU and the Cosmos may attain stability.

Critical density: This parameter is the value of the density of matter in the Universe; this implies a flat Universe, without expansion or contraction [35]. Such parameter seems very extravagant for this reason: if it has a very high value, the gravitational force is strong and the universe collapses, and if it is weak the universe accelerates. This doesn't seem to make much sense. Why would the universe accelerate when the density is low and gravity weak? What would make it accelerate? In GOP model this concept doesn't seem to make sense, the IU (Universe) is always flat, it is limited by GPW. 
Cosmic microwave background: The spectrum obtained by the satellite COBE in 1992, shows a spectrum that corresponds to the radiation of an ideal Black Body. It was also verified that there are isotropy and, sometimes, anisotropies [36]. Fluctuations in temperature were detected in privileged directions [37-39]. These have been attributed to initial disturbances before the formation of galaxies. The study of these variations is very important to Cosmology. To explain these anisotropies the models based on normal matter were discarded. Likewise, the observed $\mathrm{T} / \mathrm{T}$ fluctuations are much superior to the ones foreseen with the Cold Dark Matter model [40]. Maybe the GOP model may give some justification for this radiation and to the found anisotropies. If the Cosmic Microwave Background (CMB) corresponds to the one of a Black Body, why not think that our Universe is inside a Black Body? If we look from our Universe to the GPW its limits resemble the interior of a black body, where walls show variation in density $[41,42]$.

If $\mathrm{CMB}$ originates in this wave, we expect that it doesn't present anisotropies according to rotations with a fixed angle, in relation to the plane of the IU. If the rotation is on the plane of IU, some anisotropy may be introduced because this is the one that contains more cosmic structures. In rotations on a perpendicular direction to the plane of our Universe, the GPW are getting more and more rarefied along a 90-degree angle. In this angular variation we should expect anisotropies.

Composition of the cosmos: The Cosmos is probably only constituted by Matter $\leftrightarrow$ Energy as the persistence of laws of conservation suggests; if something more exists, either it interferes with Matter $\leftrightarrow$ Energy and the laws of conservation are compromised, or it doesn't interfere and it must be found in another universe - not in this one - what finally means that this one is just Matter $\leftrightarrow$ Energy [43]. In this model, speaking of DM and DE doesn't make sense, because it only deals with conventional matter and energy. All the phenomena now seem to be easily explained by the existence of gravitational field created by GPW in the IU.

Variation of the composition of the cosmos: The values found for the variation of the conventional matter passed from $14 \%$ at the beginning of the Cosmic Era to 4.6\% today [40]; a logical explanation for this fact hasn't been found yet. When using the proposed model, this above variation corresponds to the matter captured by the GPW since the beginning of the Cosmic Era. If GPW is in expansion, the Universe Inside will be becoming more rarefied, either by the increased of the interior space or by the fact that the GPW continue to attract and capture matter.

Absence of anti-matter in the inner universe: We know from the laboratory experiments that whenever a particle of Matter is created, particles with opposite electrical load are also created, and exactly in the same number, which are called antiparticles. Experimentally the positron or positive electron and the anti-proton or negative proton have already been created. The standard model of physics of the particles defines: The laws of physics must be equal for Matter and AntiMatter. This symmetry is important enough. This principle suggests that the Cosmos must be constituted by half of matter and by half of anti-matter. In the presence of one another, these particles annihilate themselves and they produce great quantities of energy $\left(\mathrm{E}=\mathrm{mc}^{2}\right)$. If at the beginning Matter and Anti-Matter existed in equal quantities, what was the process that led to a predominance of Matter in our Universe?

The matter and anti-matter annihilate themselves and they produce energy. This energy is associated with the particles and it provokes strong collisions among them and they give origin, again, to matter and anti-matter. At the beginning of the cosmic era these continuous processes of formation and annihilation of matter and anti-matter, due to any slight difference between them, may have led to the domination of the Matter. The GOP model allows a hypothesis: like in the atomic bomb, there is a cloud over the wave with the shape of a mushroom, there is also a cloud beyond GPW. This cloud may be formed by antimatter that, for a slight difference in temperature in relation to matter, made the anti-matter deviate itself, because it gained a greater speed. This greater speed threw the anti-matter beyond GPW, which made this become a barrier, not allowing it to attain the inner universe. This zone of anti-matter will be today very rarefied and extremely cold, and, being so, very little anti-matter destroyed by chock with GPW. Because of these conditions, it may also find itself in a non-developed state, like primordial origins of the cosmic era.

Form of the cosmic structures: The complex structures of the universe have, in general, flattened shape as the example of the galaxies classified in three types; two of them, the most significant ones present this form The elliptical galaxies occur preferably in dense zones in the interior of the clusters [44]. This is in concordance with GOP model, as in the interior, the local gravity can attenuate the effect of the GPW field and allow structures which are not plane. The Filaments and the "Walls" are also flat. The transversal cuts present a bigger and a smaller axis and the bigger axis can be aligned with the plane of the IU [20]. All this is compatible with GOP model, and it would also be predictable, because these complex structures are stretched along the plane of bigger intensity, than the one of the GPW field which is precisely the plane where our Universe is situated.

Symmetry of nebulae: Nebulae present, with frequency, a high level of symmetry. This is something mysterious. The symmetry of certain nebulae may not be more than the symmetry of the GPW field where they are situated. The less dense areas of the nebulae tend to dissipate by action of the GPW field that when competing with the local field, impose on it, with frequency, a symmetry. If the plan or axis of symmetry of the nebulae is so, it must be aligned with the lines of strength which pass by the center of the mass of the nebulae. Probably the nebulae further away from the mass center of the structure to which they belong have shapes with more strong symmetry.

Shape of the inner universe: The IU is situated in the plane of more intensity of the GPW field. We can compare it to an elastic membrane, under tension, permanently stretched by the ring GPW. All the complex structures of the Inner Universe, subject to this tension, can acquire a flattened or ellipsoid shape if the local field doesn't overlap. This happens with many galaxies, nebulae, filaments and walls.

\section{Discussion}

\section{Adjustment cosmological parameters by GOP model}

Cosmological constant: The Cosmological Constant was introduced by Einstein, because a static Universe was the most acceptable hypothesis. Einstein proposed a static, homogeneous and isotropic Universe [45]. When he applied these conditions to the Theory of Relativity, he had to add one more term, so that the Universe didn't go expanding or contracting infinitely and was kept static.

This constant needs to be thought in the light of the new conditions imposed by this model. In this model the region of the Cosmos to which this constant is applied is the IU. The value of the total mass is not now constant. The density of the IU varies from the BB in direction to GPW, thus, the universe is isotropic. The cosmological constant which seems to be a potential energy must now be seen as a gravitational potential [46-48]. 


\section{Hubble constant}

Hubble law: the galaxies move away from us with a radial speed which is proportional to our distance and which is equal in every direction. This law would allow one to determine the rhythm of the expansion of the Universe. The velocity and distance are directly proportional, being the constant known as the "Hubble Constant". This value must be reviewed, because this "constant" must square of the distance, because we are in a gravitational field.

\section{Redshift}

In this parameter, one can't consider constant the distance between the observer and the source, because most probably they dislocate themselves with different speeds. The biggest value of this difference is verified when they are aligned with the line forces of the field GPW and this difference decreases, being minimum or null when the source and the observer meet over the lines of gradient zero.

\section{Conclusion}

This model needs that the experts are interested in it and that they test it in a more accurate way and with all the problems that haven't been tested yet with any model. However, the answer given to the most crucial problems augurs a good response to the new approaches. If this model is accepted by Cosmologists, and I hope that someone will be interest in it, I want to believe that the Cosmology may know a new direction.

Knowledge is in a permanent change. We must, therefore, look without prejudice towards new ideas. This work was undertaken out of love for Scientific knowledge and, certainly, looking for a reality. May the Grandeur and Beauty of the Cosmos be an inspiration to our life, here, on Earth.

\section{References}

1. Alpher RA, Herman RC (2001) Genesis of the Big Bang. Oxford University Press. New York, USA.

2. Heisenberg W, Salam A, Dirac P (1991) Em Busca da Unificação Gradiva Publicações. Portugal.

3. Hooper D, Baltz EA (2008) Strategies for determining the nature of dark matter. Annu Rev Nucl Part Sci 58: 293-314.

4. Ferreira PG (2007) O Estado do Universo. Fundamentos Coleção 18.

5. Hawking $S$ (2006) The theory of everything: The origin and fate of the universe. Phoenix Books, Burlington, Ontario, Canada.

6. Hawking S, Mlodinow L (2012) The grand design. Reprint Edition.

7. Hawking S (1998) A brief history of time. |. Bantam Dell Publishing Group, NY, USA.

8. Hawking S (2001) O Universo Numa Casca de Noz Editora Mandarim São Paulo.

9. Hawking SW, Page DN (1983) The large scale structure of the universe. Cambridge University Press, Cambridge UK

10. Haynes R (2010) The Big Bang. Journal of Cosmology.17: 7293-7312.

11. Haynes R (2011) Zero Kelvin Big Bang an Alternative Paradigma: I. Lugic and the Cosmoic Fabric. Jounal of Cosmology.17: 7255-7278.

12. Kragh $H$ (1996) Cosmology and controversy: The historical development of two theories of the universe. Princeton University Press. Princeton, NJ, USA

13. Vieira HS, Bezerra VB (2016) Cosmologia newtoniana com Constante Cosmológica Departamento de Física Universidade Federal de Paraíba.

14. Gsponer A (2005) The physics of antimatter induced fusion and thermonuclear explosion. Cornell University Library, USA.

15. Guth $A H$ (1997) The inflationary universe. Reading MA Addison-Wesley, Boston, Massachusetts, USA.

16. Guth $\mathrm{AH}$ (1987) Inflationary universe: A possible solution to the horizon and flatness problems. Phys Rev D 23: 347-356.
17. Gibson CH, Schild RE (2011) Is dark energy falsifiable'. Journal of Cosmology.17: 7345-7358.

18. Frieman JA, Turner MS, Huterer D (2008) Dark energy and the accelerating universe. Annu Rev Astron Astrophys. 46: 385-432.

19. Drexler J (2008) Discovering postmodern cosmology. Universal-Publishers, Irvine, California, USA

20. Bharadwaj S, Bhavsar SP, Sheth JV (2004) The size of the longest filaments in the universe. Astrophysical Journal 606: 25-31.

21. FermiLab (2000) Astronomers find wall of galaxies traversing the Hubble Deeo field. DARPA.

22. Kirshner RP (2004) The extravagant universe. Princeton University, NJ, USA.

23. Gibson $\mathrm{CH}$, Nieuwenhuizen TM, Schild RE (2011) Why are so many primitive stars observed in the Galaxy Halo. Journal of Cosmology.16: 6824-6831.

24. Hubble E, Humason ML (1931) The velocity-distance relation among extragalactic nebulae. Astrophysical Journal 74: 43

25. Markevitch M, Randall S, Clowe D, Gonzalez A, Bradac M (2006). Dark matter and the bullet cluster. $36^{\text {th }}$ COSPAR Scientific assembly. Beijing, China.

26. Samuel N (2005) General Astronomy/Basic Astrophysics. School Books.

27. Peebles PJE (1993) Principles of physical cosmology. Princeton University Press, NJ, USA.

28. Peebles PJE, Ratra B (2003) The cosmological constant and dark energy. Rev Mod Phys 75: 559-606.

29. Dressler AM (1994) Voyage to the great attractor: exploring intergalactic space. Knopf. New York, NY, USA.

30. Ananthaswamy A (2010) Galaxy flow hints at huge masses over cosmic horizon. Astrophysical Journal Letters.

31. Coveney P, Roger HR (1992). The arrow of time. Ballantine Books, NY, USA.

32. Kolbb E, Turner M (1994) The early universe (Frontiers in Physics). Westview Press, Boulder, Colorado, USA.

33. Liddle AR, David HL (2000) Inflación Cosmológica y Estructura a Gran Escala Cambridge University Press, Cambridge, UK.

34. Davis T, Grifffen B (2010) Scolarpedia. 5: 4473.

35. Harrison E (2000) Cosmology - The science of the universe. Cambridge University Press. Cambridge UK.

36. Dodelson S (2003) Modern cosmology. (1st edn), Academic Press, Elsevier The Netherlands.

37. Anninos P, Matzner R, Tuluie R, Centrella J (1991) Anisotropies of the cosmic background radiation in a hot dark matter universe. Astrophys $\mathrm{J} 382: 71-78$.

38. Kovac J, Leitch EM, Pryke C, Carlstrom JE, Halverson NW, et al. (2002) Detection of polarization in the cosmic microwave background using DASI. Nature 420: 772-787.

39. Krasinski A (1996) Inhomogeneous cosmological models. Cambridge University Press, Cambridge, UK

40. Cole P, Luchin F (2002) Cosmology: The origin and evolution of cosmic structure. John Wiley \& sons, NY, USA.

41. Miller AD, Caldwell R, Devlin MJ, Dorwart WB, Herbig T, et al. (1999) A measurement of the angular power spectrum of the cosmic microwave background from I = 100 to 400 . Astrophysical Journal 524: L1-L4.

42. Mukhanov V (2005). Physical foundations of cosmology. University Press Cambridge, UK.

43. Green B (2005) The fabric of the cosmos. Vintage publishers, UK.

44. Binney J, Merrifield M (1998) Galactic astronomy. Princeton University Press, NJ, USA

45. Albert E (2008) Sobre la Teoria de la Relatividad Especial y General. Alianza Editorial, ES. Miguel Paredes Larrucea (traductor).

46. Padmanabhan T (2003) Cosmological constant - The weight of the vacuum Physics Reports 380: 235-320.

47. Penzias AA, Wilson RW (1965) Cosmological constant - The weight of the vacuum. Physics Reports. 380: 235-320.

48. Souza RE (2004) Introdução à Cosmologia EDUSP São Paulo, USA. 